\title{
Influence Model of User Behavior Characteristics on Information Dissemination
}

\author{
S.C. Han, Y. Liu, H.L. Chen, Z.J. Zhang
}

\author{
ShaoChun Han \\ Beijing Jiao tong University \\ Beijing, 100044, China \\ 15620009060@126.com
}

\section{Yun Liu}

Beijing Jiao tong University

Beijing, 100044, China

liuyun@bjtu.edu.cn

\author{
HuiLing Chen* \\ 1. School of Pharmaceutical Science and Technology Tianjin University \\ 2. TianJin University of Traditional Chinese Medicine \\ *Corresponding author: 15022613010@163.com
}

\section{ZhenJiang Zhang}

Beijing Jiao tong University

Beijing, 100044, China

zhjzhang1@bjtu.edu.cn

\begin{abstract}
Quantitative analysis on human behavior, especially mining and modeling temporal and spatial regularities, is a common focus of statistical physics and complexity sciences. The in-depth understanding of human behavior helps in explaining many complex socioeconomic phenomena, and in finding applications in public opinion monitoring, disease control, transportation system design, calling center services, information recommendation. In this paper,we study the impact of human activity patterns on information diffusion. Using SIR propagation model and empirical data, conduct quantitative research on the impact of user behavior on information dissemination. It is found that when the exponent is small, user behavioral characteristics have features of many new dissemination nodes, fast information dissemination, but information continued propagation time is short, with limited influence; when the exponent is big, there are fewer new dissemination nodes, but will expand the scope of information dissemination and extend information dissemination duration; it is also found that for group behaviors, the power-law characteristic a greater impact on the speed of information dissemination than individual behaviors. This study provides a reference to better understand influence of social networking user behavior characteristics on information dissemination and kinetic effect.
\end{abstract}

Keywords: SIR, behavior dynamics, scaling laws, information dissemination.

\section{Introduction}

The analysis target of user behavior time characteristics is the statistical regularities manifested when humans repeatedly engaged in certain things, which was firstly proposed by Poisson introduced the concept of probability in his work of case judgment management, namely Poisson distribution. When human data collection capabilities are limited, the Poisson distribution is widely used as a classic means to depict human activity patterns.

In recent years, with the emergence of high-performance processors and constant enhancement of computer parallel computing power, making the massive social network data processing 
become possible. At present, through empirical analysis, research and mining user behavior characteristics of large data and use simulation technology [1]- [8], a large number of scholars make analysis of network relationships and identify potential objective law.

By analyzing massive data of various networks, more and more facts have proven that, user behavior corresponded time interval distribution has obvious heavy-tailed effect, which can be well fitted by power function [16]- [18].

At the same time, scholars use massive data of social networks, from many fields, multi-angle and multi-dimensional human behavior characteristics were studied. For example, X.Song et al. [9] analyzed the geographical distribution of Twitter users, user's neighbor nodes and the degree of correlation coefficient, and Twitter users were grouped. H.Kwak et al. [10] studied the average shortest interval and length of Twitter micro blog, posts survival time, maximum repost depth and user grouping sorting features, the text sorted Twitter users according to the number of fans and Page-Rank value, the final results of the two sorting methods are substantially the same, which is obviously different from the final sorting result obtained by users information forwarding number, indicating that there is not tight dependencies between users information forwarding number and their owned neighbor node. M.Cha et al. [11] by comparing correlation coefficient of Twitter users posts forwarded number, post reply number and the number of neighbor nodes, studied the effect of core users on information dissemination.

The article [12] conducted further analysis of Twitter posts forwarded relevant factors. The article [13] also conducted data analysis of scholarly articles downloads from an economy physics web site, and found that download rate of different papers show exponential decrease per unit time, and the average download rate $f$ and its variance approximately satisfy $f \propto o^{\alpha}$, of which, $\alpha$ is located between 0.6 to 0.9 .

The paper [14] extracted sina blog user's interaction data, through network degree distribution analysis, it can be found that in the sina blog, the in-degree and out-degree obeys power-law distribution, but the exponent of out-degree is larger than in-degree, which explains that part of the blog user do not add more friends, and even of users do not have friends, and studies have found that the correlation coefficient of blog network in-link and out-link degree distribution is positive; while the correlation coefficient of out-link and in-link degree distribution is negative.

In this paper, firstly carry out mathematical statistical analysis of users publish information and information reply time intervals in QQ space data set, and by means of SIR [15] model to investigate influence of time characteristics of user behavior on information dissemination process in the social network, and make comparative analysis of even stepping model.

\section{Data}

In this paper, the author utilized QQ spatial data set. This data set is obtained by the use of crawling program wrote by python language. The program logins QQ space by the way of simulated browser, automatically go to access to interaction information between user and his friends, and write into the corresponding $\mathrm{xml}$ file, then read $\mathrm{xml}$ into the database by using python parsing, remove user hidden QQ number and other abnormal data, to get the final data set of QQ space. Topological statistics characteristics of the data set is shown: V denotes the number of nodes(4800), E denotes border coefficient(66475), d is the network diameter(5), $\mathrm{C}$ is network clustering coefficient(0.423), $K_{\max }$ is the maximum node degree(854), k is average node degree(30.882). 


\subsection{All nodes interval features}

All nodes features refers to the overall behavior features of all nodes in data set. Figure 1 is analysis of group posting behavior characteristic in each board; Figure 2 is analysis of group reply behavior characteristics in each board. It can be found from the figures that, all group behaviors are consistent with power-law distribution, and power exponent can be obtained through simulation fitting. In posting behavioral characteristics analysis,the exponent of message board is the largest, reaching 1.1223, while the exponent of talk board is only 0.816 , with exponent of group posting and log board is at the average;however, in analysis of reply behavioral characteristics, the exponent of talk board is the largest, is 1.1041, and the smallest is exponent of log board; so even posting and replying behavioral characteristics are different in the same board.

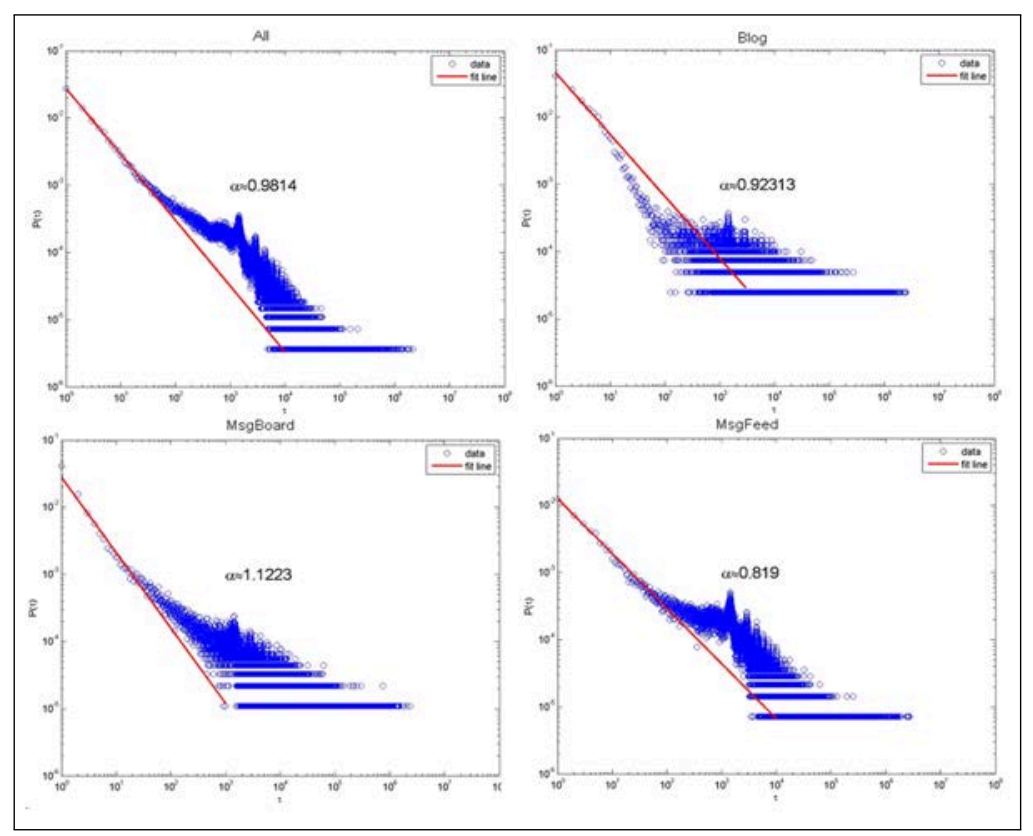

Figure 1: Analysis of group posting behavior characteristic in each board

\subsection{Individual node interval features}

The paper also analyzes the features of individual behavior, firstly the data sets are sorted in accordance with the number of nodes posting and the number of reply's in descending order, and then select the first rank (denoted by a), three-quarters (denoted as b ), intermediate (denoted as c) and fourth (denoted as d) four-node data respectively, post and reply node behavior characteristics were analyzed.

As shown in Figure 3 and 4, the actual post and reply number are at a high level at data set node $\mathrm{a}$ and $\mathrm{b}$, so the power index of posting and reply are relatively close, which shows that for active nodes, dealing with things usually by a specific behavior pattern, which is consistent with the literature conclusions; while at data set node $\mathrm{c}$ and node $\mathrm{d}$, the actual post number is few but reply of a larger number, so causing power exponent of its post behavior is small, while power exponent of reply behavior is large. To explain this phenomenon, author of this paper used NC algorithm to calculate network binding targets of these four nodes, found that nodes a and $\mathrm{b}$ have greater binding, while the binding of node $\mathrm{c}$ and $\mathrm{d}$ are small, seen from the above exponential distribution of post and reply two types of nodes and the calculation result of $\mathrm{NC}$ index value, for node $\mathrm{a}$ and $\mathrm{b}$ such very active nodes, because of its dominant position in 


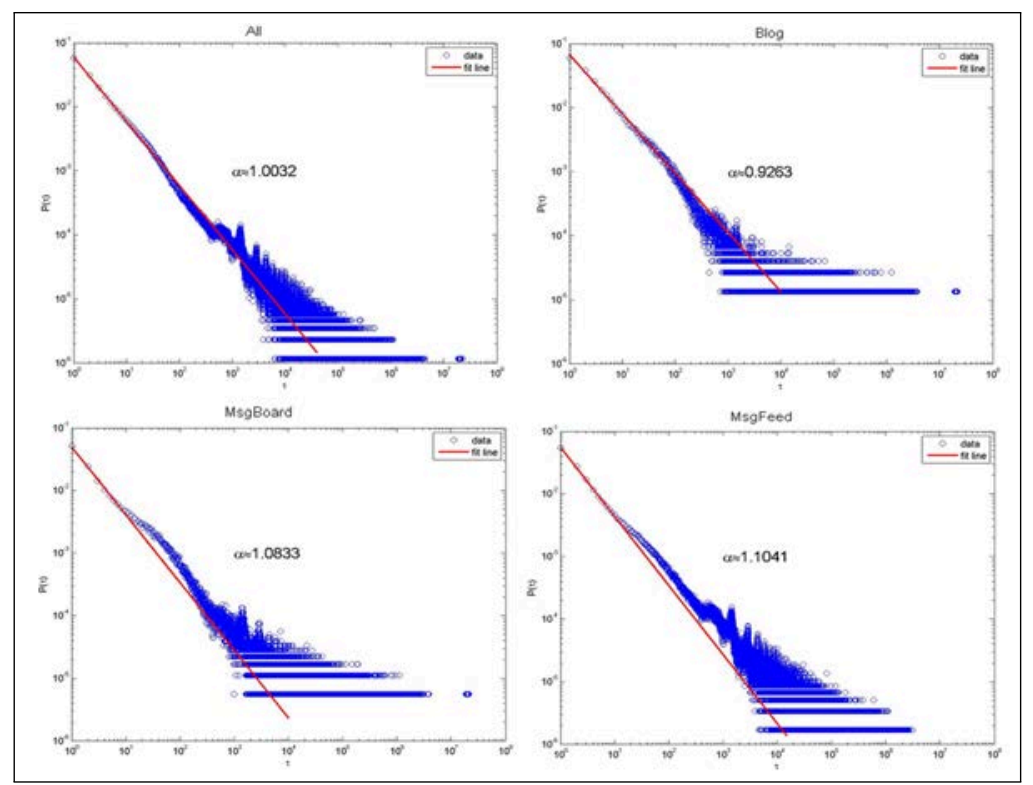

Figure 2: Analysis of group reply behavior characteristics in each board

the message, the posting content has high credibility and timeliness, resulting in follow-up reply interaction increases, while for nodes $\mathrm{c}$ and $\mathrm{d}$, as a result of information disadvantage, it can only attract other nodes to forward information by replying behavior.

Therefore, the posting and replying behaviors of active nodes are positively related, while posting and replying behaviors of non-active nodes are negatively related.

\subsection{Cluster interval features}

According to the above sort results, data sets are divided into 20 equal portions in this paper, which generate 20 clusters (the higher ranking clusters have higher degree of activity), distribution exponent of each cluster posting and reply behavior interval age calculated. In Figure 5 and Figure 6, from left to right are the eighth cluster, the tenth cluster and twelfth cluster posting and reply behavior time interval distribution. It can be found that with the lower degree of cluster activity, power index of the three cluster posting and reply behaviors also decreased, Figure 7 is exponential distribution figure of 20 cluster posting behavior, from curve trend of the figure, we can draw a conclusion that cluster activity degree is positively correlated with power exponent,which is consistent with analysis results of the literature.

\subsection{BM phase diagram analysis}

When the time interval between incidents follows power-law distribution, means that many events will concentrated occur in a relatively short period of time, followed by a long idle period, this situation is called event paroxysmal feature. From incident time interval distribution, for system with strong paroxysmal feature, most of the time interval will be less than the average event interval, but relatively large time interval may also occur, this phenomenon means that the standard deviation of its time distribution is relatively large. Event paroxysmal feature can be measured by variation coefficient B of time interval, as shown in Formula 1:

$$
B \equiv \frac{\frac{\sigma_{\tau}}{m_{\tau}}-1}{\frac{\sigma_{\tau}}{m_{\tau}}+1}=\frac{\sigma_{\tau}-m_{\tau}}{\sigma_{\tau}-m_{\tau}}
$$




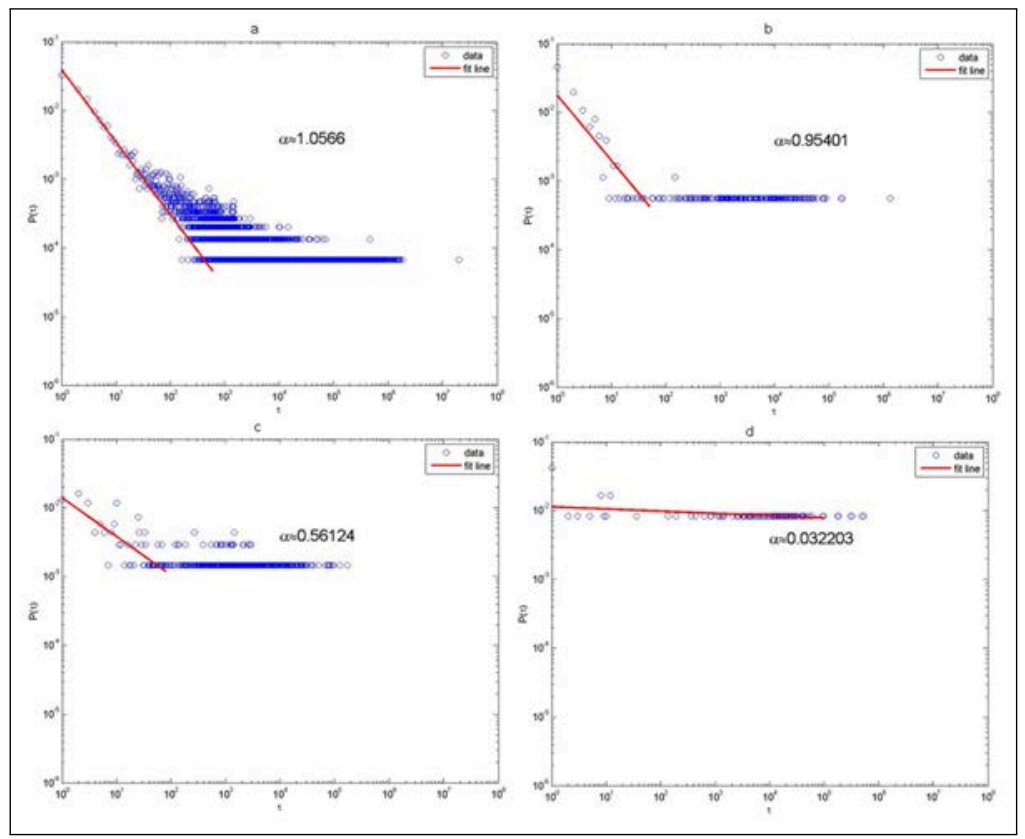

Figure 3: Characteristics of individuals posting behavior

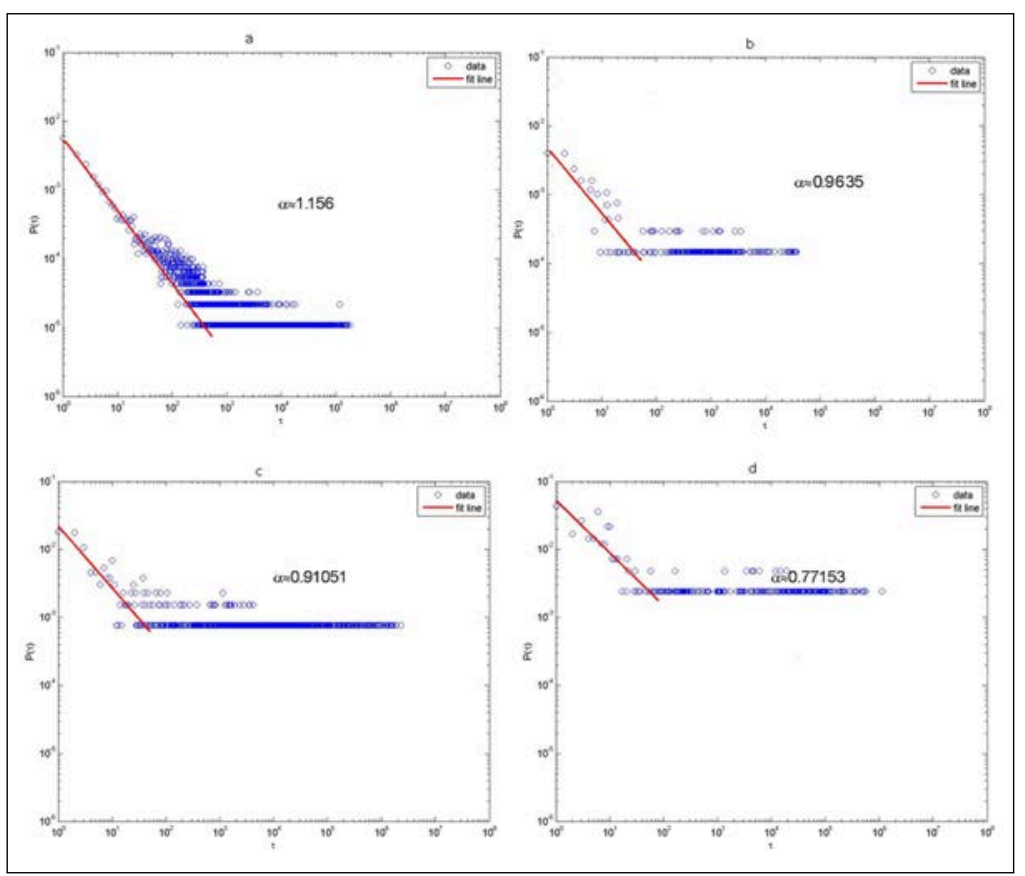

Figure 4: Characteristics of individual reply behavior

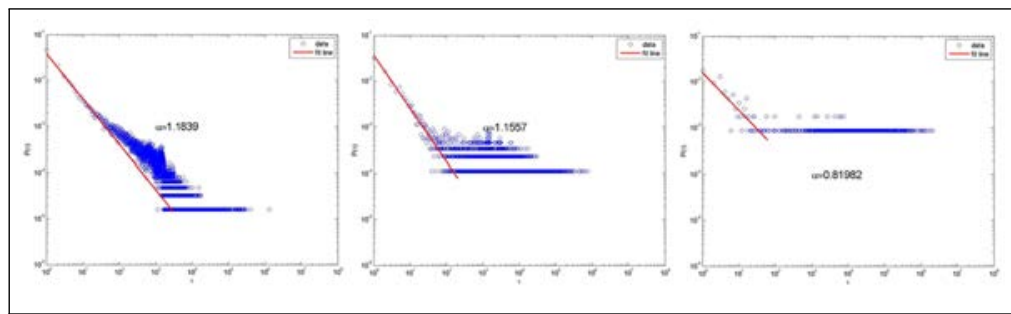

Figure 5: Analysis of three cluster post behavior characteristics 


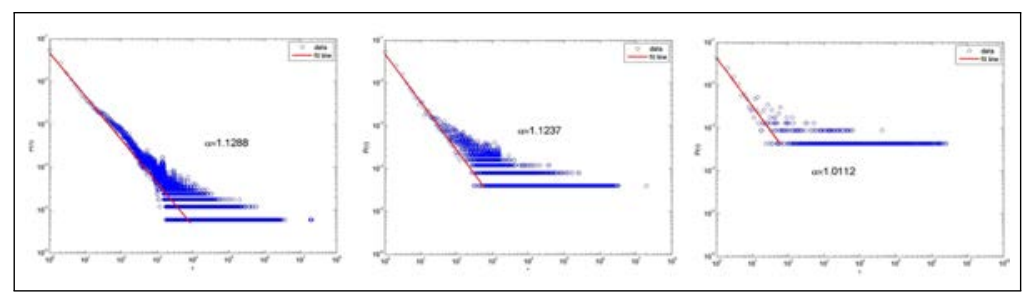

Figure 6: Analysis of three cluster reply behavior characteristics

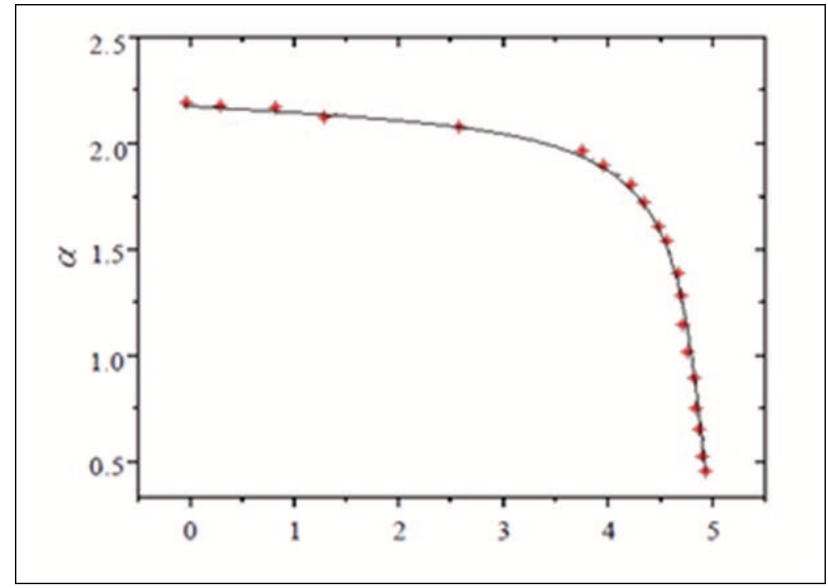

Figure 7: Relation diagram of 20 cluster exponential distribution and degree of activity

Of which $\sigma_{\tau}$ and $m_{\tau}$ represent standard deviation and average value of $p_{\tau}$, the value range of $\mathrm{B}$ is (-1 to 1$)$, with respect to the Poisson distribution, the average value and standard deviation are equal, the paroxysmal is 0 therefore, can be seen as an equilibrium point set between Goh and Barabasi; for recurring events, the time interval distribution is actually a $\delta$ function, the standard deviation is 0 , B value is -1 . For the power-law distribution, the standard deviation is much larger than average value, $\mathrm{B}$ is close to 1 , that is, the closer to 1 indicates the stronger paroxysmal, close to 0 indicates a neutral, belonging to random events series, close to -1 indicates no paroxysmal, is cyclical periodic events. In addition to paroxysmal feature of events, events characteristics may also be depicted by memory description: Time sequence of events has a certain memory, a long interval is also followed by a longer time interval, and a short interval is also followed by a shorter time interval, then all of the time intervals form a sequence according to occurring time sequence (time interval sequence of two successive behaviors), assume that this sequence has $n_{\tau}$ elements, i.e., $n_{\tau}+1$ events occurred,define the previous $n_{\tau}$-1 elements constitute sequence 1 , and define latter $n_{\tau^{-}}$elements constitute sequence two, as shown in formula 2, Pearson correlation of the two sequences can be used to measure the sequence memory.

$$
M \equiv \frac{1}{n_{\tau}-1} \sum_{i=1}^{n_{\tau}-1} \frac{\left(\tau_{i}-m_{1}\right)\left(\tau_{i}-m_{2}\right)}{\sigma_{1} \sigma_{2}}
$$

$m_{1}$ and $m_{2}$ are the mean of sequence 1 and sequence 2 respectively, $\sigma_{1}$ and $\sigma_{2}$ are standard deviation of sequence 1 and sequence 2. Obviously, the value range of $M$ is also between (-1 to 1): $M>0$ represents memory effect, $M<0$ represents anti-memory effect. When $M$ is close to 1 , indicates a long (short) time interval is more inclined to corresponding long (short) time interval after another; when closes to 0 indicates a neutral; when closes to -1 indicates a long (short) time interval is more inclined to corresponding short (long) time interval after another. Figure 8 is 


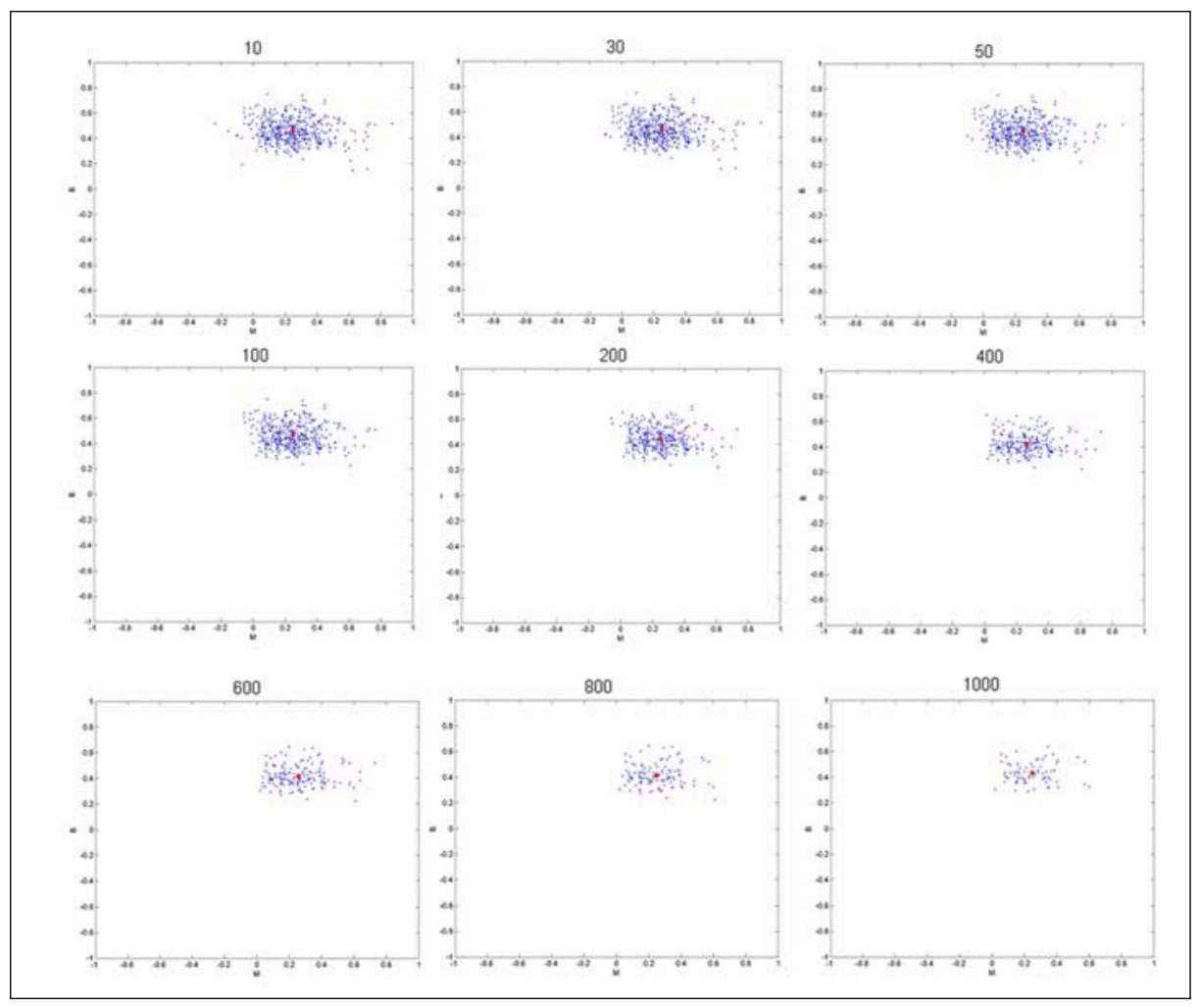

Figure 8: BM phase diagram of posting behavior

BM phase diagram of posting amount exceed 10,30, 50, 100, 200, 400, 600, 800 and 1000 posts respectively in data set. It can be found from the figure that, although there are great variance in number of posts, their average point location in BM phase diagram are stable between at the horizontal coordinates (0.244-0.26) and vertical coordinates (0.416-0.464), indicating that in QQ space, user posting behavior has obvious paroxysmal and memory features.

\section{Model building}

In this paper, the information dissemination model involved utilized SIR model of infectious disease dynamics to complete. Define users of social network as nodes, friends relationship between nodes is defined as side, and nodes are divided into three categories: Stifler,Ignorant and Spreaders. Stiflers indicates that the node receives and knows contents of the information, but it does not take the initiative to disseminate this information; Ignorant indicates that the node has not received the information sent by the neighboring nodes, so it will not conduct information dissemination, but it will receive this information at a certain probability; Spreaders indicates that the node has received information from one of its neighboring nodes, at the next timestepping, the node will take the initiative to disseminate information to its neighboring nodes. For Spreaders, in information dissemination process, if the neighbor node receiving information belongs to Ignorant, then the neighboring node may become Spreaders, and the node will conduct information dissemination at next time; if neighbor node receiving information belongs to Spreaders or Stiflers, i.e.the receiver has received the information, then the information receiving party will abandon to continue to spread this information, and if the neighbor node is Spreaders, it will change its own state into Stiflers. It can thus be seen that for social network node, its transfer between Stiflers, Ignorant and Spreaders states depends not only on the node's state at 
the current time-stepping step, also relates to state of its neighbor nodes for information interaction. At the same time this model assumes: Social network structure is relatively static, that is number of nodes, sides relationship and weight between nodes do not change with time; in the social network, node send messages to its neighbor nodes at certain time-stepping,and after neighbor node receiving the information, it will be forwarded at a certain degree of probability;information will only spread among neighbors. To sum up, let the state transition rules as follows:

1. Infection probability $\lambda$ : After Spreaders sending message to its neighbor nodes, Ignorant will become Spreaders at $\lambda$ probability, and this information will be disseminated at the next time-stepping;

2. Stiflers probability $\mu$ : Spreaders will become Stiflers at $\mu$ probability, if and only if Spreaders contact with other neighbors Spreaders or Stiflers;

3. Attenuation probability $v$ : with time attenuation, Spreaders will no longer take the initiative to disseminate information, so define attenuation probability $v$, namely, when Spreaders has no interaction with any neighbor nodes, it will automatically become Stiflers at $v$ probability.

The probability $\lambda$ of Ignorant $\mathrm{i}$ to believe and actively disseminate information is associated with its neighbor node spread influence, so the spread probability of node i is:

$$
\lambda=1-\prod_{j \in \phi_{i}}\left(1-\frac{\omega_{i j}}{\sum_{m=1}^{k_{i}}} \omega_{i j}\right)
$$

Where in $\phi_{i}$, is defined as the set of spread nodes of neighbor nodes to node i, $\omega_{i j}$ is defined as the connection weight between node i and node $\mathrm{j}$. According to SIR propagation model, it shows the entire information dissemination process:

$$
\begin{gathered}
\text { Spreaders }(j)+\operatorname{Ignorant}(i) \stackrel{\lambda}{\rightarrow} \operatorname{Spreader}(j)+\operatorname{Spreaders}(i) \\
\operatorname{Spreaders}(m)+\operatorname{Spreaders}(n) \stackrel{\mu}{\rightarrow} \operatorname{Stiflers}(m)+\operatorname{Spreaders}(n) \\
\operatorname{Spreaders}(m)+\operatorname{Stiflers}(k) \stackrel{\mu}{\rightarrow} \operatorname{Stiflers}(m)+\operatorname{Stiflers}(k) \\
\operatorname{Spreaders}(p) \stackrel{v}{\rightarrow} \operatorname{Stiflers}(p)
\end{gathered}
$$

\subsection{Propagation model}

According to the evolution rule of Formula 3, build mean-field differential evolution equations of information dissemination model. Define S, I and R to represents Spreaders, Ignorant and Stiflers states; for nodes of $\mathrm{k}$ degree, define the total number of nodes in Spreaders, Ignorant and Stiflers states as $M_{K, S}, M_{K, I}$ and $M_{K, R}$, the total number of all nodes k degree is $M_{K}$, and define:

$$
M_{K}=M_{K, S}+M_{K, I}+M_{K, R}
$$

Assuming in social network, the node I is in Ignorant state at $\mathrm{t}$ time, in $[\mathrm{t}, \mathrm{t}+\Delta t]$ period of time, define $p_{I I}^{i}$ as the probability of i will remain in Ignorant state, define $p_{I S}^{i}$ as the probability of $\mathrm{i}$ to change from Ignorant state to Spreaders state, $\operatorname{and} p_{I S}^{i}=1-p_{I I}^{i}$. If $\mathrm{j}$ remains in Ignorant state in $\triangle t$ period of time, indicating that the neighbor node of $\mathrm{j}$ in the Spreaders state fail to disseminate information to $\mathrm{j}$. So $p_{I I}^{i}$ can be expressed as:

$$
p_{I I}^{i}=\prod_{m=0}^{g}\left(1-\triangle t \lambda^{i}\right)
$$


Where in $\mathrm{g}=\mathrm{g}(\mathrm{t})$ is defined as the total number of nodes neighboring $\mathrm{i}$ at $\mathrm{t}$ time, specifically as follows:

$$
\prod g, t=\phi(k, t)^{g}(1-\phi(k, t))^{k-g}
$$

Where in, $\phi(k, t)$ is defined as probability of Ignorant of $\mathrm{k}$ degree has adjacency relationship with certain Spreaders at t time:

$$
\prod(g, t)=\sum_{k^{\prime}} P\left(K_{\prime}^{\prime} \mid k\right) P\left(S_{k^{\prime}} \mid I_{k}\right) \approx \sum_{k \prime} P\left(K_{\prime}^{\prime} \mid k\right) \rho^{S}(k \prime, t)
$$

In formula $8, P\left(K^{\prime} \mid k\right)$ is the correlation function of degree, that is conditional probability of node of $\mathrm{k}$ degree and node of $K^{\prime}$ degree have adjacency relationship; $P\left(S_{k \prime} \mid I_{k}\right)$ is defined when node of $K$ / degree has connection relationship with uninfected node of $\mathrm{k}$ degree, the node belongs probability of propagation state; $\rho^{s}(k \prime, t)$ is defined as Spreaders density at t time and $K \prime$ degree. By traversing all possible $\mathrm{g}$ and $\mathrm{i}$, the mean of $p_{I I}^{i}$ can be calculated, i.e. $\bar{p}_{I I}(k, t)$, which is used to describe the mean field dynamics equation of the model:

$$
\bar{p}_{I I}(k, t)=\frac{1}{M_{k}} \sum_{i} \sum_{g=0}^{k} \prod_{m=0}^{g}\left(1-\Delta t \lambda^{m i}\right) \phi(k, t)^{g}[1-\phi(k, t)]^{k-g}
$$

Where in, $M_{k}$ is the total number of all nodes of $\mathrm{k}$ degree in the network. In $[\mathrm{t}, \mathrm{t}+\triangle t]$ period of time, $p_{S S}^{i}$ is defined as the probability of Spreaders to maintain spread state, traverse all possible $\mathrm{g}$, the average probability $\bar{p}_{I I}(k, t)$ for node to maintain spread state:

$$
\begin{aligned}
\bar{p}_{I I}(k, t) & =\sum_{g=0}^{k}(1-\mu \triangle t)^{g} \phi(k, t)^{g}(1-\phi(k, t))^{k-g}(1-v \triangle t) \\
& =\sum_{g=0}^{k}(1-\mu \triangle t) \phi(k, t)^{g}(1-\phi(k, t))^{k-g}(1-v \triangle t) \\
& =(1-\mu \triangle t) \phi(k, t)+1-\phi(k, t)^{k}(1-v \triangle t) \\
& =\left(1-\mu \triangle t \phi(k, t)^{k}\right)(1-v \triangle t) \\
& =\left(1-\mu \triangle t \sum_{k \prime} P(K \prime \mid k)\left[\rho^{S}(k \prime, t)+\rho^{R} s(k \prime, t)\right]\right)^{k}(1-v \triangle t)
\end{aligned}
$$

The probability for Spreaders to become Stiflers (contact) can be expressed as $\bar{p}_{S R}(k, t)=$ $1-\bar{p}_{S S}(k, t)$. On the basis of average state transition probability, according to node change rules of formula 5 , changing situation of nodes of $\mathrm{k}$ degree in three states in $[\mathrm{t}, \mathrm{t}+\Delta t]$ time period can be obtained, as shown in Equation 11, 12 and Equation 13:

$$
\begin{aligned}
M_{K, I}(t+\triangle t) & =M_{K, I}(t)-M_{K, I}(t)\left(1-\bar{p}_{I I}(k, t)\right) \\
& =M_{K, I}(t)-M_{K, I}(t)\left[1-\frac{1}{M_{k}} \sum_{i} \sum_{g=0}^{k} \prod_{m=0}^{g}\right. \\
& \left.\left(1-\triangle t \lambda^{m i}\right) \phi(k, t)^{g}[1-\phi(k, t)]^{k-g}\right]
\end{aligned}
$$




$$
\begin{gathered}
M_{K, I}(t+\triangle t)=M_{K, S}(t)+M_{K, I}(t)\left(1-\bar{p}_{I I}(k, t)\right)-M_{K, S}(t)\left(1-\bar{p}_{S S}(k, t)\right) \\
=M_{K, S}(t)+M_{K, I}(t)\left[1-\frac{1}{N_{k}} \sum_{i} \sum_{g=0}^{k} \prod_{m=0}^{g}\left(1-\triangle t \lambda^{m i}\right)\right. \\
\left.\phi(k, t)^{g}[1-\phi(k, t)]^{k-g}\right]-M_{K, S}(t)\left[1-\left(1-\mu \triangle t \sum_{k \prime} P\left(K^{\prime} \mid k\right)\right.\right. \\
\left.\left.\left[\rho^{S}(k \prime, t)+\rho^{R} s(k \prime, t)\right]\right)^{k}(1-v \triangle t)\right] \\
M_{K, R}(t+\triangle t)=M_{K, R}(t)+M_{K, S}(t)\left(1-\bar{p}_{S S}(k, t)\right) \\
=M_{K, R}(t)+M_{K, S}(t)\left[1-\left(1-\mu \triangle t \sum_{k \prime} P\left(K_{\prime}^{\prime} \mid k\right)\right.\right. \\
\left.\left.\left[\rho^{S}(k \prime, t)+\rho^{R} s(k \prime, t)\right]\right)^{k}(1-v \triangle t)\right]
\end{gathered}
$$

To simplify the calculations, denote $\Phi(k, g, t)=\phi(k, t)^{g}(1-\phi(k, t))^{k-g}$, For Formula 11, the following variants can be realized:

$$
\frac{M_{K, I}(t+\triangle t)-M_{K, R}(t)}{M_{K}}=\frac{M_{K, I}(t)}{M_{K}}\left[1-\frac{1}{M_{k}} \sum_{i} \sum_{g=0}^{k} \prod_{m=0}^{g}\left(1-\triangle t \lambda^{m i}\right) \Phi(g, k, t)\right]
$$

Denote $\rho^{I}(k, t)=\frac{M_{K, I}(t)}{M_{K}}$. Both ends of Formula(14)take $\triangle t \rightarrow 0$.

Since $\lim _{\triangle t \rightarrow 0} \prod_{m=0}^{g}\left(1-\triangle t \lambda^{m i}\right)=\sum_{m=0}^{g}\left(-\lambda^{m i}\right)$, so Formula (14) can be defined as:

$$
\frac{\varphi \rho^{I}(k, t)}{\varphi(t)}=\frac{\rho^{I}(k, t)}{M_{K}} \sum_{j} \sum_{m=1}^{g} \sum_{g=1}^{k} \Phi(g, k, t) \lambda^{m i}
$$

Similarly, it can be derived from Formula (13)

$$
\begin{aligned}
\frac{M_{K, R}(t+\triangle t)-M_{K, R}(K, t)}{N_{K}} & =\frac{M_{K, S}(K, t)}{N_{K}}\left(1-\bar{p}_{S S}(K, t)\right) \\
& =\frac{M_{K, S}(K, t)}{m_{K}}\left[1-\left(1-\mu \triangle t \sum_{k \prime} P(K \prime \mid k)\right.\right. \\
& {\left.\left.\left[\rho^{S}(k \prime, t)+\rho^{R} s(k \prime, t)\right]\right)^{k}(1-v \triangle t)\right] }
\end{aligned}
$$

At right end of Formula(16)

$$
\begin{aligned}
& \left(1-\mu \triangle t \sum_{k \prime} P\left(K_{\prime}^{\prime} \mid k\right)\left[\rho^{S}(k \prime, t)+\rho^{R} s(k \prime, t)\right]\right)^{k} \\
& =\sum_{k=0} n C(k, n)\left(-\mu \triangle t \sum_{k \prime} P\left(K_{\prime}^{\prime} \mid k\left[\rho^{S}(k \prime, t)+\rho^{R} s(k \prime, t)\right]\right)^{n}\right. \\
& =C_{0}^{K}+C_{1}^{K}\left(-\mu \triangle t \sum_{k \prime} P\left(K^{\prime} \mid k\left[\rho^{S}(k \prime, t)+\rho^{R} s(k \prime, t)\right]\right)\right. \\
& =1-k \mu \triangle t \sum_{k^{\prime}} P\left(K^{\prime} \mid k\left[\rho^{S}(k \prime, t)+\rho^{R} s(k \prime, t)\right]\right.
\end{aligned}
$$

Therefore, Formula(16) is varied: 


$$
\begin{aligned}
& \frac{M_{K, R}(t+\Delta t)-M_{K, R}(K, t)}{N_{K}} \\
& =\rho^{S}(k, t)\left[1-\left(1-k \mu \triangle t \sum_{k \prime}\right.\right. \\
& \left.\left.P\left(K_{\prime}^{\prime} \mid k\right)\left[\rho^{S}(k \prime, t)+\rho^{R} s(k \prime, t)\right]\right)(1-v \triangle t)\right] \\
& =\rho^{S}(K, t)\left(k \mu \triangle t \sum_{k \prime} P\left(K^{\prime} \mid k\right)+v \Delta t+k \mu \triangle t^{2} \sum_{k \prime}\right. \\
& \left.P\left(K^{\prime} \mid k\right)\right)\left[\rho^{S}(k \prime, t)+\rho^{R}(k \prime, t)\right]
\end{aligned}
$$

Both ends of Formula (18) divide $\triangle t$ and take the limit $\triangle t \rightarrow 0$.

$$
\frac{\varphi \rho^{R}(k, t)}{\varphi(t)}=k \mu \rho^{S}(k, t) \sum_{k \prime}\left[\rho^{S}(k \prime, t)+\rho^{R}(k \prime, t)\right] P(k \prime \mid k)+v \rho^{S}(k, t)
$$

Since $\frac{\varphi \rho^{I}(\alpha, k, t)}{\varphi(t)}+\frac{\varphi \rho^{S}(\alpha, k, t)}{\varphi(t)}+\frac{\varphi \rho^{R}(\alpha, k, t)}{\varphi(t)}=0$,so it is easy to obtain:

$$
\begin{aligned}
\frac{\varphi \rho^{S}(k, t)}{\varphi(t)} & =\frac{\rho^{I}(k, t)}{M_{k}} \sum_{j} \sum_{m=1}^{g} \sum_{g=1}^{k} \Phi(g, k, t) \lambda^{m i} \\
& -k \mu \rho^{S}(k, t) \sum_{k \prime}\left[\rho^{S}(k \prime, t)+\rho^{R}(k \prime, t)\right] P(k \prime \mid k)-v \rho^{S}(k, t)
\end{aligned}
$$

By Formula (15), (19) and (20) simultaneous,obtain the dynamical evolution equations of information dissemination in social network for depicting changes in the relationship between Spreaders,Ignorant and Stiflers density over time.

\section{Simulation analysis}

Figure 9 is the propagation and evolution diagram of Stiflers, Ignorant and Spreaders density under different power exponent $\alpha$ and even time-stepping $(\alpha=0)$, and from left to right are Spreads density evolution diagram, Ignorant evolution diagram and Stiflers evolution diagram. It can be seen from Spreads density evolution diagram that, with the decrease of power exponent $\alpha$, peak of wave of Spreads density continues to decline, but the final information dissemination duration has been prolonged, indicating that the smaller heterogeneity of temporal characteristics of user behaviors, breadth of information dissemination may be affected, but the final information dissemination duration will be extended. It can be seen from Ignorant evolution diagram and Stiflers evolution diagram that, for the former, with the decrease of power exponent $\alpha$, the greater proportion of Ignorant; while for the latter, with the decrease of power exponent $\alpha$, the smaller proportion of Stiflers.

Figure 10 is respectively represent evolution diagram of the proportion of new Spreaders number per time step and proportion of cumulative Spreaders number, which also confirmed from edgewise that the power-law characteristic of user activity time distribution will have a huge impact on information dissemination. When the power exponent is small, although there are many new Spreaders, the information dissemination comes and goes fast, which may not have a greater impact; but when the power exponent is large, although the number of people implementing information dissemination is small, it lasts longer, it will produce more lasting influence, which explains the phenomenon why some information gets spread again after a long silence.

Relaxation time refers to the time required for model to start from evolution to tended to be 


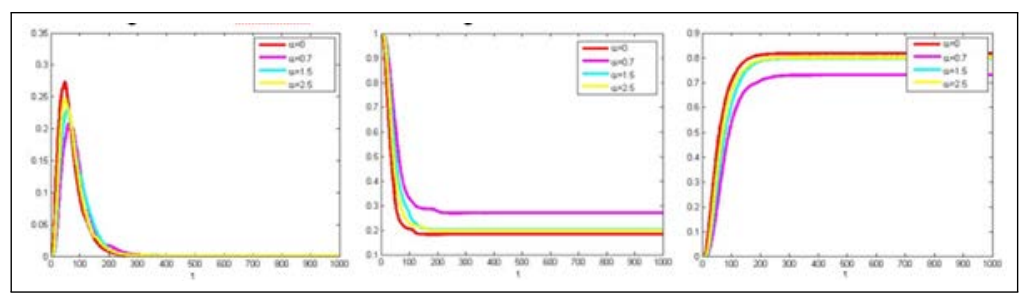

Figure 9: Influence of time-order character on propagation
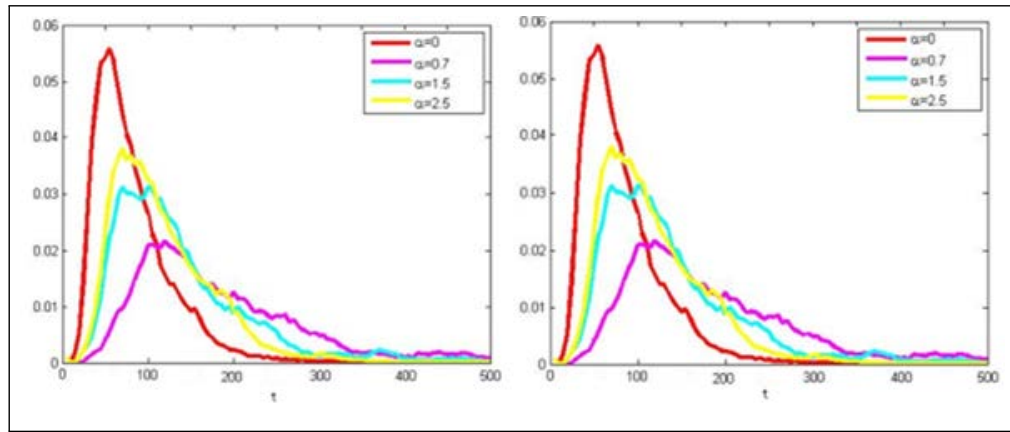

Figure 10: Left:Evolution diagram of proportion of new Spreaders number per time step. Right: Evolution diagram of proportion of cumulative Spreaders number

stable, Figure 11 are the relationship between power exponent and the relaxation time and respectively represent when the number of Spreads goes over half of time t, It can be seen from the figure that, as the power exponent increases, the relaxation time shows obvious linear downward trend. Since the power-law distribution of user behavior can be expressed in group and individual nodes, so the paper conducted simulation analysis of impact of power-law characteristic at groups and individual-level on information dissemination process. The so-called power-law distribution at group level refers to the behavior time distribution sequence of each node is regular, namely the time interval of node behavior remains unchanged,but the degree of activity between nodes varies greatly, and meets power-law distribution. Power-law distribution at individual-level refers to the time interval distribution of individual's own behavior meets the power-law characteristic, but the degree of activity and time intervals satisfied different distribution between individuals are the same.

The center and right respectively represent when the number of Spreads goes over half of time t, impact of power-law distribution characteristics group and individual level on speed of information dissemination relationship diagram. It can be seen from the above figures that, at the group level, the power-law distribution has greater impact on the dissemination of information, while power-law distribution at individual level has less effect on the dissemination of information.

Figure 12 respectively represent relation diagram between the maximum propagation range of information dissemination $C_{\max }$ and the maximum node propagation density $S_{\max }$ and power exponent, as it can be seen from the figure,with continued exponential increases, the maximum propagation range of information dissemination $C_{\max }$ and $S_{\max }$ the maximum node propagation density simultaneously increase, but when the power index $\alpha>1.5$, the maximum propagation range of information dissemination has been stabilized, while it will still have some impact on the maximum node propagation density $S_{\max }$. 


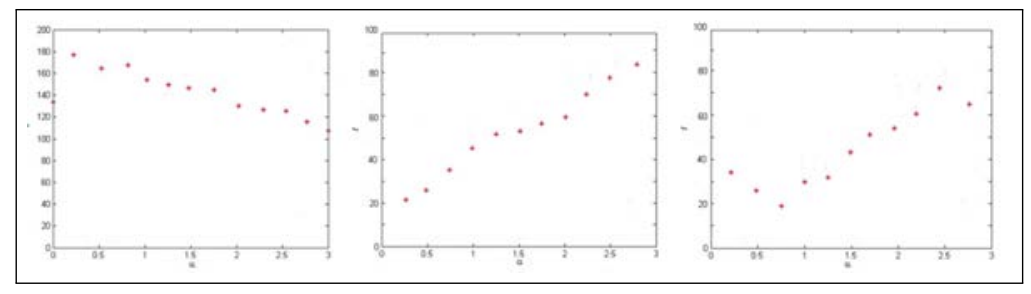

Figure 11: Left:Relationship between power exponent $\alpha$ and relaxation time. Center:Impactof power-law distribution characteristics group-level on speed of information dissemination. Right: Impact of power-law distribution characteristics individual-level on speed of information dissemination

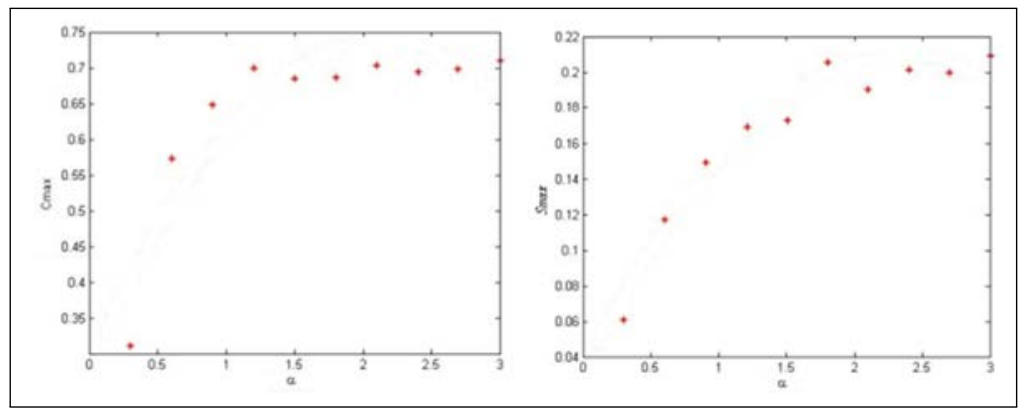

Figure 12: Left:Relation diagram between the maximum propagation range of information dissemination and power exponent. Right: Relation diagram between the maximum node propagation density and power exponent

\section{Conclusion}

With empirical data of social networks, here conduct quantitative analysis of user behavior time interval characteristics, which is conducive to explain many complex networks information propagation phenomena, and can generate social benefits and application value in the public opinion monitoring, disease prevention, information recommendation and other aspects. Firstly, the paper makes use of real social network user behavior data, to analyze information posting and reply behaviors of networking groups, network individuals and network groups respectively, then use the BM phase diagram to analyze paroxysmal and memory characteristics of user's information posting and reply behaviors. By means of SIR propagation model and empirical data, implement quantitative study of impact of user behavior time interval characteristic on information dissemination process, and found that user behavior time interval meets the characteristics of power-law distribution, although it will slow speed of information dissemination to a great extent, it will also extend the duration of the information dissemination and can increase the ultimate scale of information dissemination, and thus more likely to have a greater impact on the society; It also found that at the group level, power-law characteristic has a greater impact on speed of information dissemination, while at individual level, the speed of information dissemination is less affected by power-law characteristic.

\section{Acknowledgment}

This research is supported by:

1. National Natural Science Foundation of China (No.61271308);

2. Beijing Natural Science Foundation (No.4112045); 
3. Beijing City Science and Technology Project (No.Z121100000312024);

4. Specialized Research Fund for the Doctoral Program of Higher Education of China (No. W11C100030).

\section{Bibliography}

[1] Zhou, T.; Han, X.P.et al(2013); Statistical Mechanics on Temporal and Spatial Activities of Human, Journal of University of Electronic Science and Technology of China, ISSN 1674862X, 4(4):481-540.

[2] Zhang, H.P.(2015); An agent-based simulation model for supply chain collaborative technological innovation diffusion, International Journal of Simulation Modelling, ISSN 1726-4529, 14(2):313-324.

[3] Liu, S.; Gong,D.(2014); Modelling and simulation on recycling of electric vehicle batteriesusing agent approach, International Journal of Simulation Modelling, ISSN 1726-4529, 13(1):79-92.

[4] Pasztor, A.(2014); Gathering simulation of real robot swarm, Technical Gazette, ISSN 18486339, 21(5):1073-1080.

[5] Shang, Y.l.(2013); Measuring degree-dependent failure in scale-free networks of bipartite structure, International Journal of Simulation 83 Process Modelling, ISSN 1740-2131, 8(1):7478.

[6] Lerher, T.; Ekren, Y.B.; Sari,Z.;Rosi,B.(2015); Simulation Analysis of Shuttle Based Storage and Retrieval Systems, International Journal of Simulation Modelling, ISSN 1726-4529, 14(1):48-59.

[7] Cho, Y.C.(2015); A novel approach of adaptive socially aware routing algorithm in delay tolerant networks, Technical Gazette, ISSN 1848-6339, 22(1):61-70.

[8] Xue, Y.G.et al(2014); Determination of statistical homogeneity by comprehensively considering the discontinuity information, Technical Gazette, ISSN 1848-6339, 21(5),971-977.

[9] Java, A.; Song, X.; Finin, T.; Tseng,B.(2007); WebKDD/SNAKDD 2007:web mining and social network analysis post-workshop report, Acm Sigkdd Explorations Newsletter, 9(2):8792.

[10] Kwak, H.; Lee, C.; Park, H.(2010); What is Twitter,a Social Network or a News Media,International conference on World wide web,591-600.

[11] Cha, M.; Haddadi, H.et al(2010); Measuring user influence in Twitter: the million follower fallacy, Proceedings of the Fourth International AAAI Conference on Weblogs and Social Media, 23-26.

[12] Suh, B.; Hong, L.; Pirolli, P.; Chi, E.H.(2010); Want to be Retweeted? Large Scale Analytics on Factors Impacting Retweet in Twitter Network, 2010 IEEE Second International Conference on Social Computing, 177-184.

[13] Han, D.D.et al(2008); Fluctuation of the Download Network, Chinese Physics Letters, ISSN 0256-307X, 25(2):765-768. 
[14] Fu, F.; Liu, L.H.; Wang, L.(2008); Empirical analysis of online social networks in the age of Web 2.0, Physica A, ISSN 0378-4371, 387(2):675-684.

[15] Wang, Z. et al(2015); Coupled disease-behavior dynamics on complex networks: A review, Physics of Life Reviews, ISSN 1571-0645, 15(1):30-31.

[16] Alessandro, A.; Laura, B.; George, L.(2015); Privacy and human behavior in the age of information, Science, 347(6221):509-14.

[17] Freitas, C.R.D.(2015); Weather and place-based human behavior: recreational preferences and sensitivity, International Journal of Biometeorology, ISSN 0020-7128, 59(1):55-63.

[18] Medina, J.R.; Lorenz,T.; Hirche, S.(2015); Synthesizing Anticipatory Haptic Assistance Considering Human Behavior Uncertainty, Robotics IEEE Transactions on, 31(1):180-190. 